\title{
Communication \\ Wide-Field-of-View Near-Eye Display with Dual-Channel Waveguide
}

\author{
Chao Ping Chen ${ }^{1}\left(\mathbb{D}\right.$, Yuepeng Cui ${ }^{2}$, Yuning Ye ${ }^{1}$, Feiyang Yin ${ }^{1}$, Huiwu Shao ${ }^{1}$, Yan Lu ${ }^{1}\left(\mathbb{D}\right.$ and Gang Li ${ }^{1,3, *(\mathbb{D})}$ \\ 1 Smart Display Lab, Department of Electronic Engineering, Shanghai Jiao Tong University, \\ Shanghai 200240, China; ccp@sjtu.edu.cn (C.P.C.); 1956860113@sjtu.edu.cn (Y.Y.); y25625@sjtu.edu.cn (F.Y.); \\ 652shw@sjtu.edu.cn (H.S.); cosmic_ly@sjtu.edu.cn (Y.L.) \\ 2 Shanghai InfoCrops Science \& Technology Co., Ltd., Shanghai 201802, China; albert@infotek.com.cn \\ 3 School of Psychology and Neuroscience, University of Glasgow, Glasgow G12 8QB, UK \\ * Correspondence: gang.li@glasgow.ac.uk
}

check for updates

Citation: Chen, C.P.; Cui, Y.; Ye, Y.; Yin, F.; Shao, H.; Lu, Y.; Li, G. Wide-Field-of-View Near-Eye Display with Dual-Channel Waveguide. Photonics 2021, 8, 557. https:// doi.org/10.3390/photonics 8120557

Received: 7 November 2021 Accepted: 6 December 2021 Published: 7 December 2021

Publisher's Note: MDPI stays neutral with regard to jurisdictional claims in published maps and institutional affiliations.

Copyright: (c) 2021 by the authors. Licensee MDPI, Basel, Switzerland. This article is an open access article distributed under the terms and conditions of the Creative Commons Attribution (CC BY) license (https:/ / creativecommons.org/licenses/by/ $4.0 /)$.

\begin{abstract}
We propose a wide-field-of-view near-eye display featuring a dual-channel waveguide with cholesteric liquid crystal gratings. Our dual-channel waveguide is capable of splitting the field of view through the orthogonal polarization division multiplexing. To explain its mechanism, a diagram of $k$-domain, which factors into both the waveguide size and the number of pupils, is depicted. Our results demonstrate that the diagonal field of view reaches up to $80^{\circ}$, eye relief is $10 \mathrm{~mm}$, exit pupil is $4 \times 3 \mathrm{~mm}^{2}$, and uniformity is $79 \%$.
\end{abstract}

Keywords: near-eye display; field of view; dual-channel waveguide; exit pupil expander; cholesteric liquid crystal grating

\section{Introduction}

In the display community, the story of near-eye displays (NEDs) [1] is dramatically inspiring. For a very long time, this type of displays was sitting quietly in the corner. It was not until a few years ago - thanks to the hype of augmented/virtual reality (AR/VR) - that suddenly NEDs were thrust into the limelight. People are starting to believe that NEDs may shift the paradigm and transform the way we see and interact with machines. Still, some might argue that it is enough to have mature flat-panel displays (FPDs) [2,3] for AR/VR and NEDs are not necessary. But what makes NEDs unique-among other traits-is something called immersion, which could improve the user experience and ramp up the productivity. To deliver immersion, a big field of view (FOV) is needed. For VR NEDs, it is not an issue. But for AR NEDs-particularly those with waveguides [4-11] -FOV is definitely a bottleneck. This is because the waveguides are inevitably engaged with the total internal reflection (TIR), whose critical angle is responsible for a significant loss in FOV. To address this matter, a number of solutions have been proposed. The most straightforward one is to increase the refractive indices of waveguides. Both Schott and Corning-two giant glass makers-have recently managed to supply the industry with AR-ready glass wafers with a refractive index over 1.9. An alternative way is to bypass the TIR by directing the light nonstop from the in-coupling grating to out-coupling grating [7]. By doing so, FOV no longer hinges on the refractive index. Yet, the downside is the incapability of exit pupil expansion (EPE). To take care of both FOV and exit pupil, Microsoft's HoloLens gen 2 introduced a butterfly-like EPE that leverages a split FOV mechanism [12]. Compared to its predecessor, its FOV jumped from $35^{\circ}$ to $52^{\circ}$ [13]. However, this design also received much criticism. Karl Guttag - a well-known AR/VR blogger-pointed out that HoloLens gen 2 suffered from poor uniformity [14]. We attribute this problem to the partial overlapping-with a shape of an inverted trapezoid - between the left and right EPE regions.

Over the course of evolution of technology, a two-step-forward-and-one-step-back situation is not uncommon. Inspired by the said contributions, we hereby present a 
waveguide-based NED, in which FOV is split unidirectionally or one-way into two channels. In the following sections, we will discuss how to design the dual-channel waveguide, and explore its performance and merits.

\section{Design Rules}

\subsection{Dual-Channel Waveguide}

Figure 1 schematically draws the cross-section of dual-channel waveguide, the top and bottom layers of which are referred to as channel 1 and channel 2, respectively. The left and right half-FOVs shall be of orthogonal polarizations, e.g., left-handed and righthanded circular polarizations. Gratings in channel 1-including the in-coupling and out-coupling-are responsive merely to the light coming from the left half of FOV. Gratings in channel 2, on the other hand, will take care of the rest of FOV, i.e., the right half. To evenly expand the pupil, the out-coupling grating consists of five sub-gratings-abbreviated as $\mathrm{O} 1 / \mathrm{O} 2 / \mathrm{O} 3 / \mathrm{O} 4 / \mathrm{O} 5$ - with their efficiencies being modulated. For the sake of big FOV, RealView glass (Schott) [15] is chosen as the material of waveguide, whose refractive index $n_{w g}$ at $546 \mathrm{~nm}$ is 1.91048 , yielding to a critical angle $\theta_{c}$ of $31.57^{\circ}$. For the light inside the waveguide to be reflected at angles greater than $\theta_{c}$, gratings of channel 1 and channel 2 are tilted by $\theta_{1}$ and $\theta_{2}$, respectively. Other physical parameters regarding the waveguide and gratings, as shown in Figure 2, can be also found in Table 1 , where $W / H / D$ is the width/height/thickness of waveguide, $W_{i / o} / H_{i / o}$ the width/height of in/out-coupling grating, and $L_{i o}$ the distance between the in-coupling and out-coupling gratings.

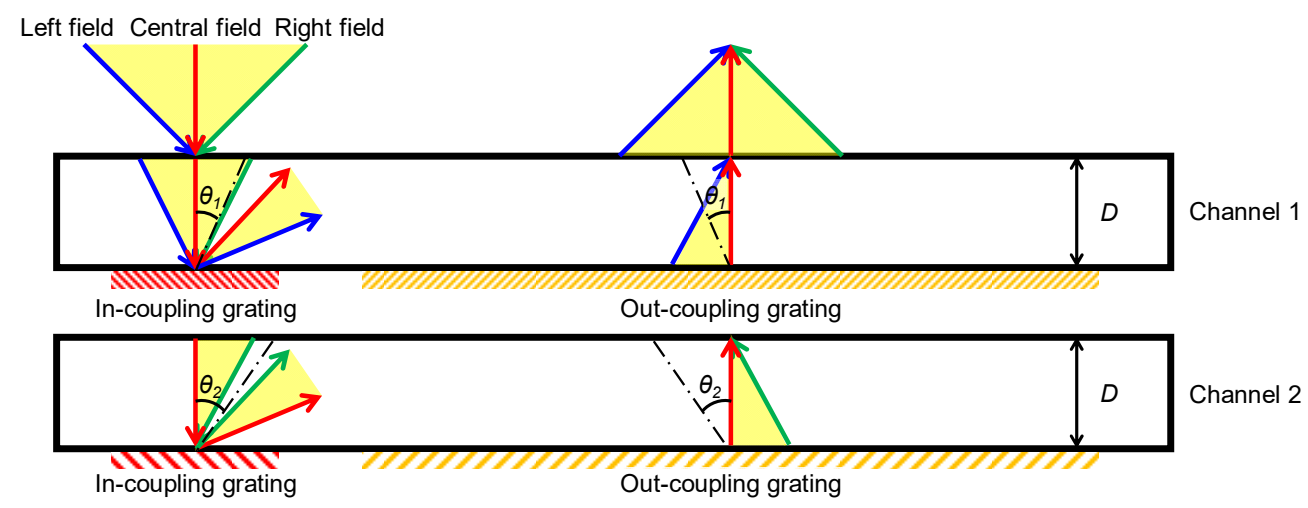

Figure 1. Cross-section of dual-channel waveguide, the top and bottom layers of which are referred to as channel 1 and channel 2, respectively. The left and right half-FOVs shall be of orthogonal polarizations, e.g., left-handed and right-handed circular polarizations. Gratings in channel 1-including both the in-coupling and out-coupling-are responsive merely to the light coming from the left half of FOV. Gratings in channel 2, on the other hand, will take care of the rest of FOV, i.e., the right half.

Table 1. Parameters of waveguide and gratings.

\begin{tabular}{ccc}
\hline Object & Parameter & Value \\
\hline \multirow{2}{*}{ Waveguide } & $W$ & $36 \mathrm{~mm}$ \\
& $H$ & $24 \mathrm{~mm}$ \\
& $n_{w g}(633 / 546 / 486 \mathrm{~nm})$ & $1.67 \mathrm{~mm}$ \\
& $\theta_{c}(546 \mathrm{~nm})$ & $1.89781 / 1.91048 / 1.92411$ \\
In-coupling grating & $W_{i}$ & $31.57^{\circ}$ \\
\hline & $H_{i}$ & $4 \mathrm{~mm}$ \\
& $\theta_{1 / 2} \mathrm{~mm}$ \\
\hline & $W_{o}$ & $16^{\circ} / 25^{\circ}$ \\
\hline & $H_{o}$ & $20 \mathrm{~mm}$ \\
& $L_{i o}$ & $12 \mathrm{~mm}$ \\
\end{tabular}




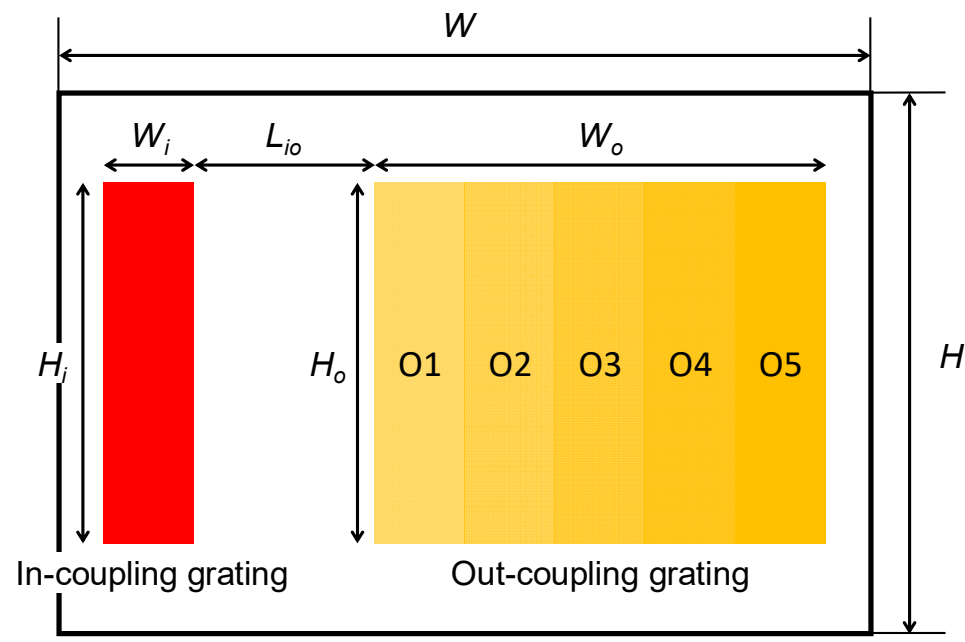

Figure 2. Plan view of dual-channel waveguide. To evenly expand the pupil, the out-coupling grating consists of five sub-gratings-abbreviated as O1/O2/O3/O4/O5—with their diffraction efficiencies being modulated. $W / H / D$ is the width/height/thickness of waveguide, $W_{i / o} / H_{i / o}$ the width/height of in/out-coupling grating, and $L_{i o}$ the distance between the in-coupling and out-coupling grating.

\subsection{Number of Pupils}

For a given field $\theta_{w g}$-measured within the waveguide-its pupil will be duplicated by the number of TIRs $N_{T I R}$, which can be determined from

$$
N_{T I R}=\frac{W_{o}}{2 D \tan \theta_{w g}}
$$

As shown in Figure 3, when $\theta_{w g}<\theta_{\mathcal{c}}\left(31.57^{\circ}\right)$, TIR does not occur. When $\theta_{w g} \geq \theta_{\mathcal{c}}$, the number of TIRs decreases all the way to 0 . Say the number of duplicated pupils is supposed to be no less than 5 . Then, the upper limit of field $\theta_{\max }$ will max out at $50^{\circ}$. Hence, $5 \leq N_{T I R}<10$ and $31.57^{\circ} \leq \theta_{\text {wog }} \leq 50^{\circ}$.

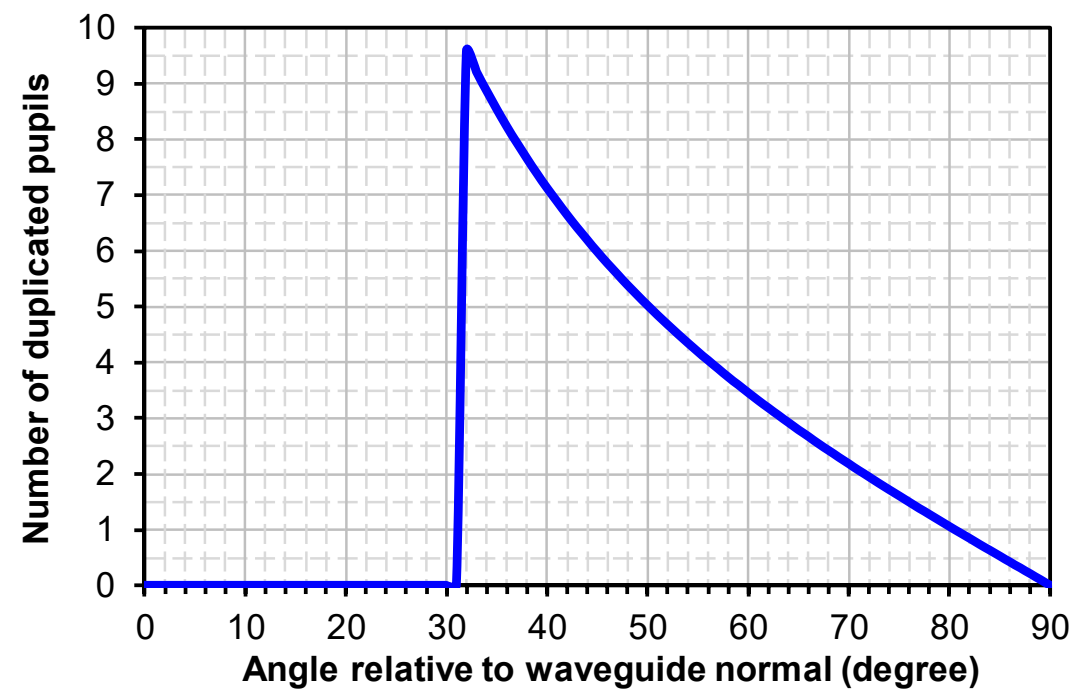

Figure 3. Number of duplicated pupils with respect to the angle relative to the waveguide normal. When $\theta_{w g}<\theta_{\mathcal{c}}\left(31.57^{\circ}\right)$, TIR does not occur. When $\theta_{w g} \geq \theta_{c}$, the number of TIRs decreases all the way to 0 . Say the number of duplicated pupils is supposed to be no less than 5 . Then, the upper limit of field $\theta_{\max }$ will max out at $50^{\circ}$. 


\section{3. $k$-Domain}

In an ideal scenario-when the waveguide can be infinitely large or curved or inhomogeneous, and minimally required number of TIRs or pupils is not considered-the theoretical boundary of $k$-domain solely depends on the refractive index of medium once the wavelength $\lambda$ is given [11]. However, for a finite, flat, homogeneous waveguide, there shall exist a practical boundary of $k$-domain that will be defined by a circle with a radius $R_{\max }$

$$
R_{\max }=\frac{2 \pi n_{w g}}{\lambda} \sin \theta_{\max }
$$

Suppose the input $\mathrm{FOV}$ is $80^{\circ}$, i.e., $72^{\circ}$ (horizontal) $\times 45^{\circ}$ (vertical), and the gratings of channel 1 and channel 2 could couple the light in and out such that:

$$
\sin ^{-1}\left(\frac{n_{\text {air }} \sin \theta_{\text {air }}}{n_{\text {wg }}}\right)+\theta_{1 / 2}=\theta_{\text {wg }}-\theta_{1 / 2}
$$

where $n_{\text {air }}$ is the refractive index of air, $\theta_{\text {air }}$ the field measured in air, and $\theta_{\text {wg }}$ the field measured in waveguide. When $\lambda=546 \mathrm{~nm}, n_{w g}=1.91048, \theta_{\max }=50^{\circ}$ (for the minimal $N_{T I R}=5$ ), and $\theta_{1 / 2}=16^{\circ} / 25^{\circ}$, $k$-domain can be plotted, as shown in Figure 4 , in which green/blue rounded rectangle denotes the left/right-half FOV, innermost circle the theoretical boundary in air, and intermediate/outermost circle the practical/theoretical boundary in waveguide. It can be seen that, after splitting, two half-FOVs are located at the same side of $k$-domain, meaning that the EPE regions of two channels could be seamlessly overlapped.

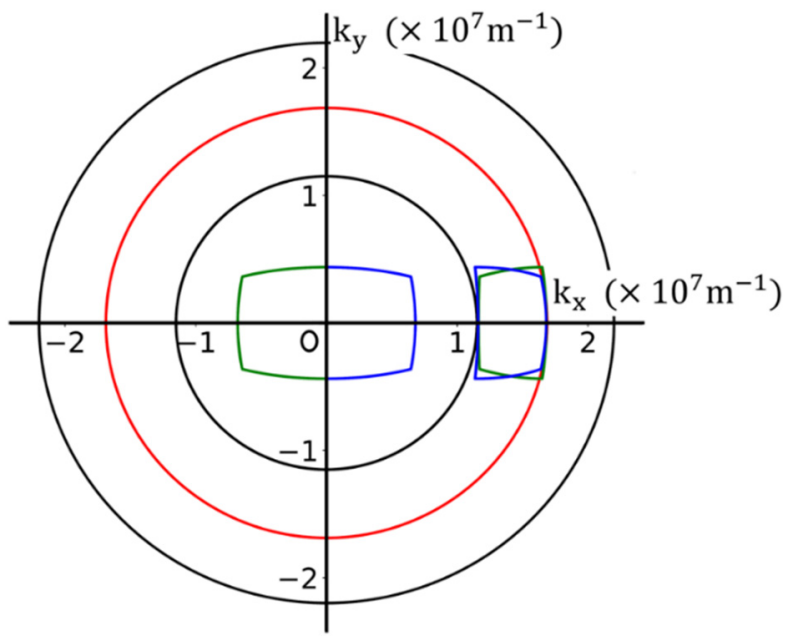

Figure 4. Diagram of $k$-domain calculated when $\lambda=546 \mathrm{~nm}, n_{w g}=1.91048$, and $N_{T I R}=5$. Green $/$ blue rounded rectangle denotes the left/right-half FOV, innermost circle the theoretical boundary in air, and intermediate/outermost circle the practical/theoretical boundary in waveguide.

\subsection{Cholesteric Liquid Crystal Grating}

In search of the gratings that would do the said jobs, we resort to the cholesteric liquid crystal (CLC) grating for several reasons. Number one, it is polarization-sensitive, i.e., only responsive to circularly polarized light of certain handedness [16]. Number two, it can be made achromatic by stacking or other multi-color techniques [17] so as to minimize the number of layers of waveguides. Since its reflection is per se a type of Bragg diffraction, the reflected wavelength $\lambda_{B}$ and incident angle $\theta_{i}$ shall be correlated via

$$
m \lambda_{B}=p \cos \theta_{i} \sqrt{\left(n_{e}^{2}+2 n_{0}^{2}\right) / 3}
$$

where $m$ is the diffraction order, $p$ the helical or chiral pitch, and $n_{e}$ and $n_{o}$ the extraordinary and ordinary refractive indices of liquid crystal, respectively. As Equation (4) indicates, 
CLC exhibits both wavelength and angular selectivity. In order for the gratings to be wide-band as well as wide-angle, a stack of CLCs satisfying different Bragg conditions are essential. Number three, CLC lends itself to waveguide couplers for its high efficiency and variable grating normal. The former can be guaranteed as long as the CLC is sufficiently thick to be a polarization volume grating. The latter is achieved by introducing a geometric or Pancharatnam-Berry (PB) phase through photoalignment [16] or holography [18] to CLC such that its grating normal can be tilted by the angle $\theta_{1 / 2}$ as [19]

$$
\sin \theta_{1 / 2}=\frac{p}{2 \Lambda}
$$

where $\Lambda$ is the PB pitch, also known as the alignment pitch. It is worth noting that Equation (5) is only valid for the slanted, thick CLC configuration. As for other possible CLC configurations, the calculation of grating normal will be subject to change [20].

\section{Results and Discussion}

\subsection{Field of View, Eye Relief and Exit Pupil}

Figure 5 shows the geometric relationship among the FOV, eye relief (ER) and exit pupil (EP). With both the grating and optical adhesive sandwiched in between being ignored and applying the trigonometry together with Snell's law, this relationship could be formulated as [11]

$$
\mathrm{EP}=W_{o} / H_{o}-3 \cdot D \cdot \tan \left(\sin ^{-1}\left(\frac{n_{\text {air }} \sin \left(\frac{\mathrm{FOV}}{2}\right)}{n_{w g}}\right)\right)-2 \cdot \mathrm{ER} \cdot \tan \left(\frac{\mathrm{FOV}}{2}\right)
$$

from which one takeaway is that a big FOV tends to have a small exit pupil. For FOV $=72^{\circ}$ (horizontal) $\times 45^{\circ}$ (vertical) and $\mathrm{ER}=10 \mathrm{~mm}, \mathrm{EP}=4 \times 3 \mathrm{~mm}^{2}$. Incidentally, in addition to scaling up the waveguide or to shortening the eye relief [21], another solution is to make the waveguide movable to accommodate the variation of interpupillary distance.

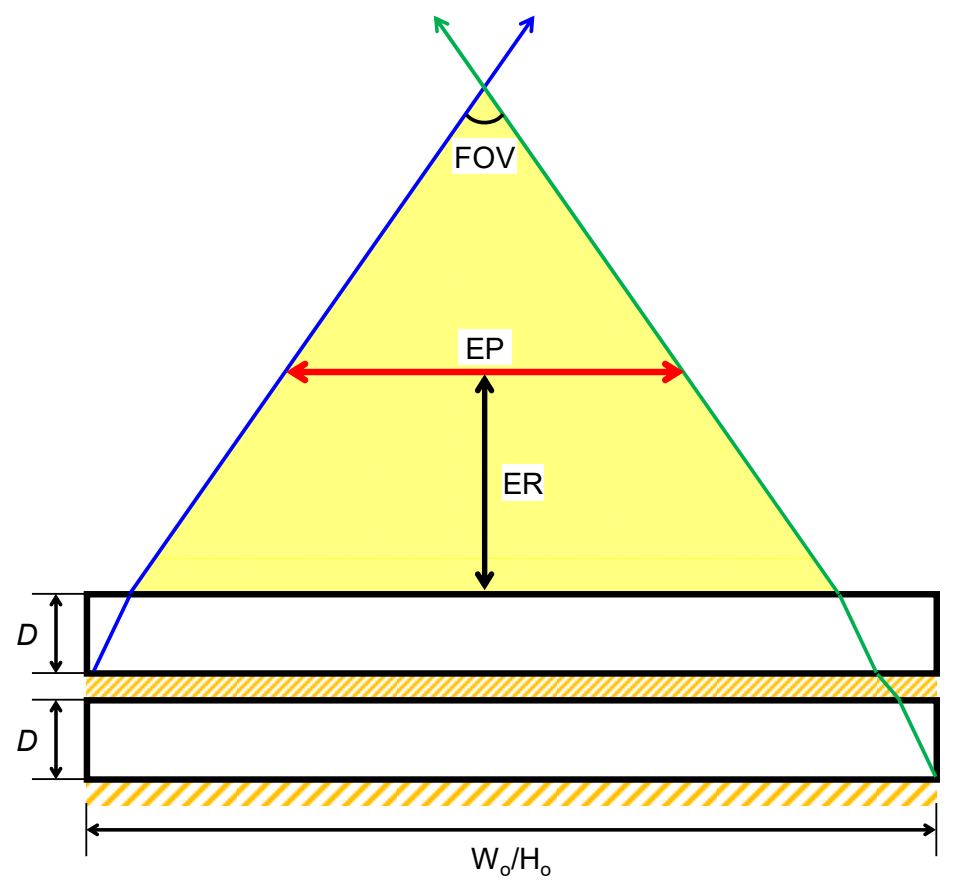

Figure 5. Geometric relationship among the FOV, eye relief and exit pupil. For FOV $=72^{\circ}$ (horizontal) $\times 45^{\circ}$ (vertical) and $\mathrm{ER}=10 \mathrm{~mm}, \mathrm{EP}=4 \times 3 \mathrm{~mm}^{2}$. 


\subsection{Grating Efficiency}

Table 2 lists the parameters for our tri-layer-stacked CLCs, whose Bragg wavelength, thickness, chiral pitch, and thickness-to-pitch ratio are specified. The calculation of stacked CLCs follows from the $4 \times 4$ matrix method [22]. As for the spectral bandwidth, the reflection efficiency or reflectance is computed with respect to the wavelength, as shown in Figure 6. The spectral bandwidth of reflectance from $45 \%$ to $50 \%$ for the red (R)/green (G)/blue (B) color is $47 / 40 / 34 \mathrm{~nm}$, which is compatible with the light-emitting diodes as the light source. As for the angular bandwidth, reflectance is computed with respect to the incident angle-measured relative to the grating normal—as shown in Figure 7 . The angular bandwidth of reflectance from $45 \%$ to $50 \%$ for R/G/B is 50/52/52 degrees, which is wide enough to cover half of the FOV.

Table 2. Parameters for stacked CLC gratings.

\begin{tabular}{cccc}
\hline CLC Layer & Bragg Wavelength & Parameter & Value \\
\hline \multirow{2}{*}{$633 \mathrm{~nm}$} & $d$ & $8 \mu \mathrm{m}$ \\
& & $p$ & $422.16 \mathrm{~nm}$ \\
& & $d / p$ & 18.95 \\
\hline \multirow{2}{*}{$\mathrm{G}$} & \multirow{2}{*}{$546 \mathrm{~nm}$} & $d$ & $8 \mu \mathrm{m}$ \\
& & $p$ & $364.30 \mathrm{~nm}$ \\
& \multirow{3}{*}{$486 \mathrm{~nm}$} & $d / p$ & 21.96 \\
\hline \multirow{2}{*}{$\mathrm{B}$} & & $d$ & $8 \mu \mathrm{m}$ \\
& & $d / p$ & $324.28 \mathrm{~nm}$ \\
& & & 24.67 \\
\hline
\end{tabular}

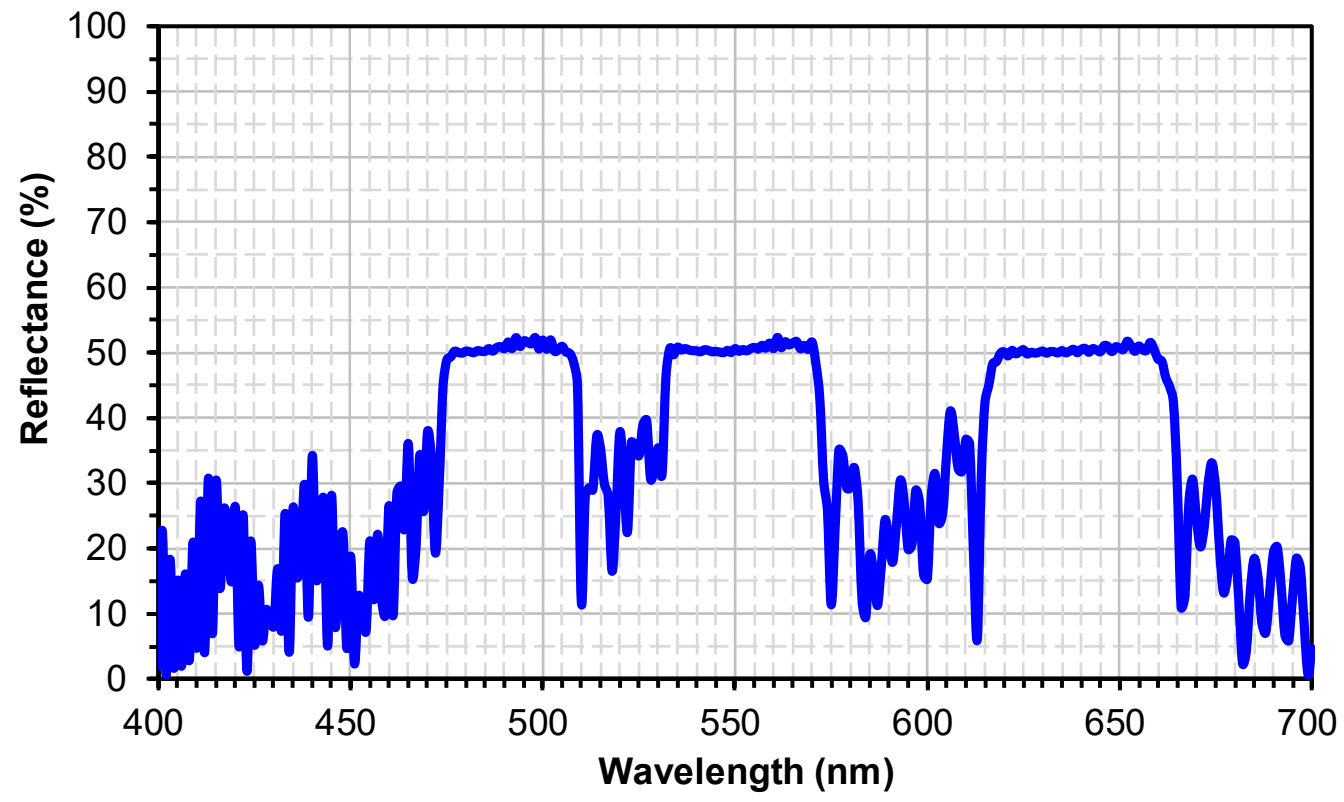

Figure 6. Reflectance with respect to the wavelength. The spectral bandwidth of reflectance from $45 \%$ to $50 \%$ for the $\mathrm{R} / \mathrm{G} / \mathrm{B}$ color is $47 / 40 / 34 \mathrm{~nm}$, which is compatible with the light-emitting diodes as the light source. 


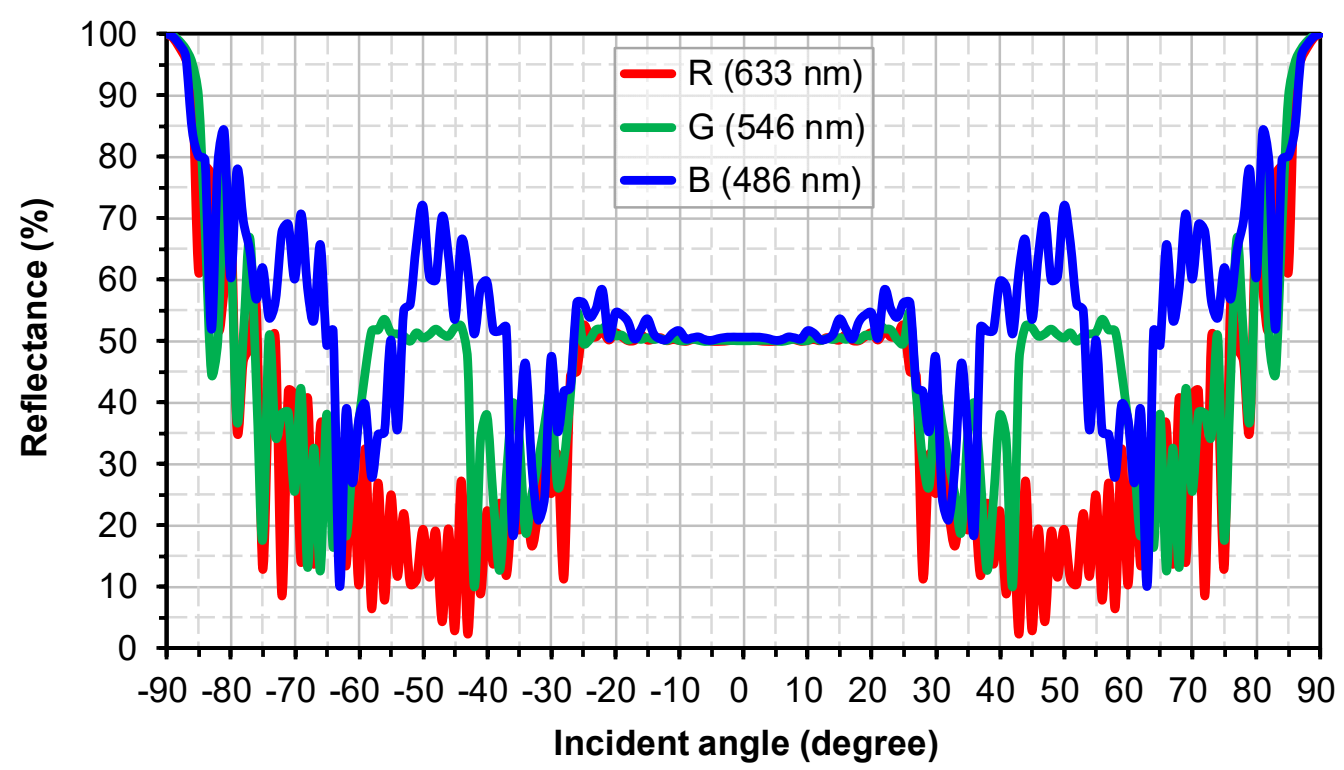

Figure 7. Reflectance with respect to the incident angle. The angular bandwidth of reflectance from $45 \%$ to $50 \%$ for $\mathrm{R} / \mathrm{G} / \mathrm{B}$ is $50 / 52 / 52$ degrees, which is wide enough to cover half of the FOV.

\subsection{Uniformity}

With VirtualLab Fusion, the location of duplicated exit pupils can be obtained by tracing the rays of central field, as shown in Figure 8, where the entrance pupil is circular and $4 \mathrm{~mm}$ across. In order for the duplicated pupils to be uniform, by setting the uniformity error of the detector as the merit function and employing the Nelder-Mead or downhill simplex method, the normalized grating efficiencies of the reflected zeroth (R0) and first (R1) orders are tweaked as in Table 3. The uniformity, defined as

$$
\text { Uniformity }=\frac{2 I_{\min }}{I_{\max }+I_{\min }}
$$

where $I_{\max }$ and $I_{\min }$ are in turn the maximum and minimum intensities, can be analyzed by calculating the electromagnetic fields at the detector, as shown in Figure 9. The overlapped regions of pupils, where the light interferes with one another, are treated with coherent summation. As $I_{\max }=0.2337 \mathrm{~V}^{2} / \mathrm{m}^{2}$ and $I_{\min }=0.1510 \mathrm{~V}^{2} / \mathrm{m}^{2}$, the uniformity is $79 \%$.

Table 3. Normalized efficiencies of gratings.

\begin{tabular}{ccc}
\hline Grating & Efficiency of R0 (\%) & Efficiency of R1 (\%) \\
\hline In-coupling & 100 & 0 \\
O1 & 89.687 & 10.313 \\
O2 & 90.260 & 9.740 \\
O3 & 89.521 & 10.479 \\
O4 & 88.285 & 11.715 \\
O5 & 72.735 & 27.265 \\
\hline
\end{tabular}




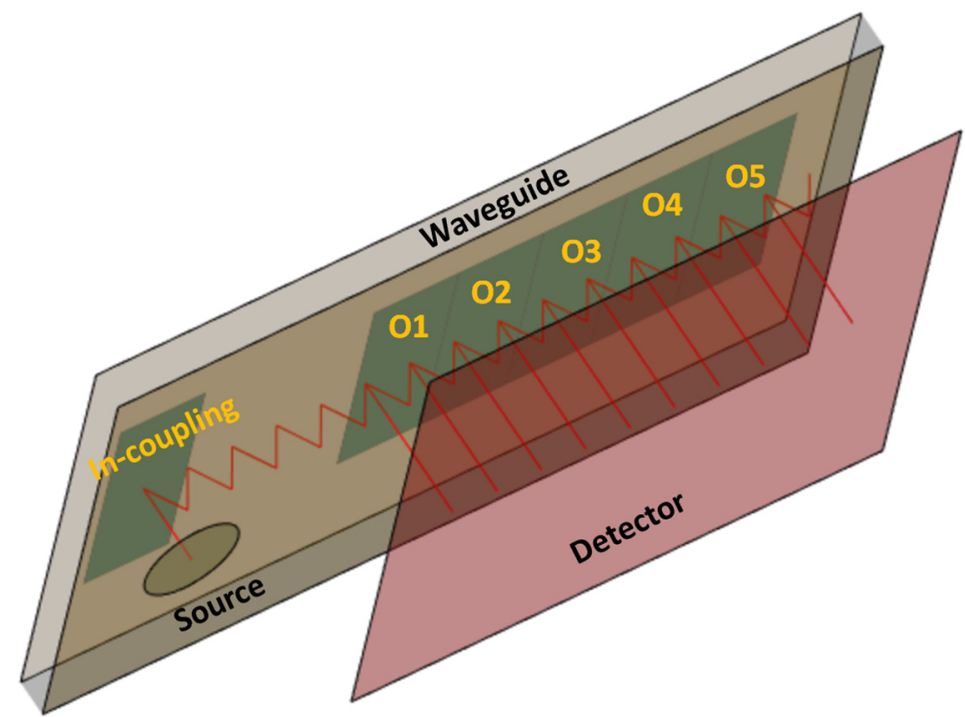

Figure 8. Location of duplicated exit pupils obtained by tracing the rays of central field. The entrance pupil is circular and $4 \mathrm{~mm}$ across.

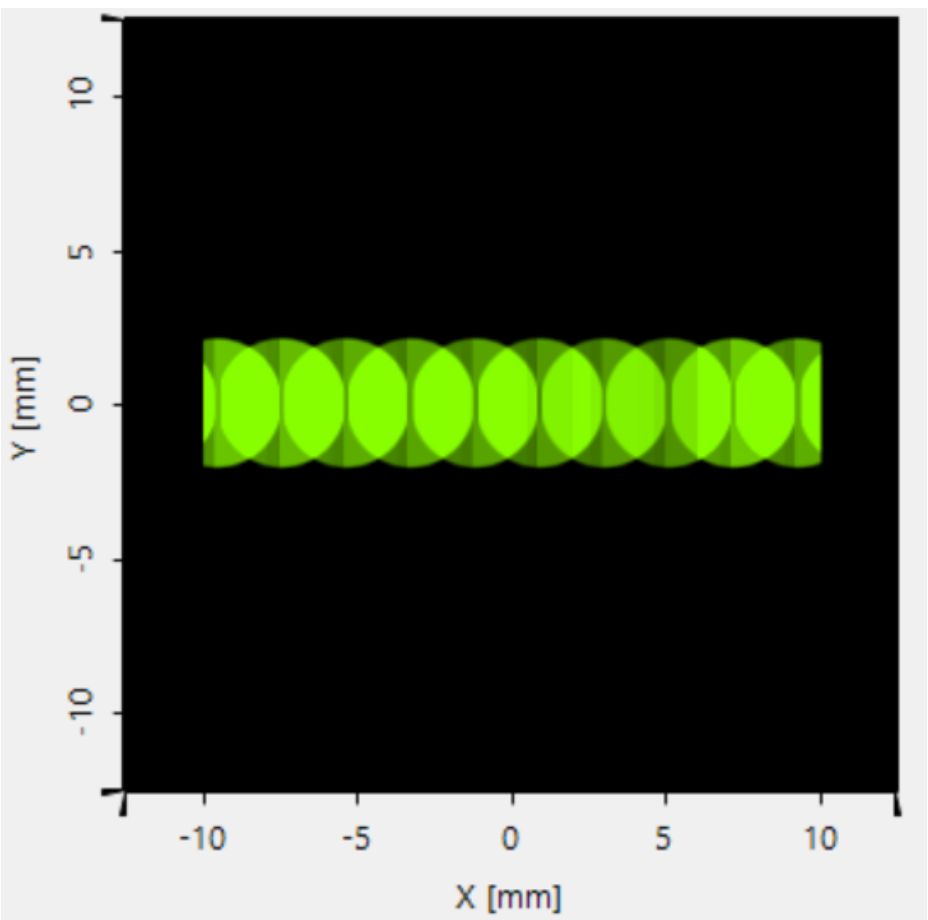

Figure 9. Uniformity analysis of duplicated exit pupils by calculating the electromagnetic fields at the detector. The overlapped regions of pupils, where the light interferes with one another, are treated with coherent summation.

\section{Conclusions}

In conclusion, a wide-field-of-view NED with a dual-channel waveguide and design rules thereof have been studied. Instead of splitting the FOV by direction [11,12], our method is to split the FOV by polarization. The most important benefit is that the EPE regions of different channels could be overlapped. This could not only maximize the waveguide real estate or active area, but also improve the uniformity. In what follows, its key performance is recapitulated. $\mathrm{FOV}$ is $80^{\circ}$ (diagonal), $\mathrm{ER}=10 \mathrm{~mm}, \mathrm{EP}=4 \times 3 \mathrm{~mm}^{2}$, and uniformity is $79 \%$. 


\section{Patents}

In light of the potential impact of this work, one invention patent (application number: 202111225152.8) was filed to China National Intellectual Property Administration on 21 October 2021.

Author Contributions: Conceptualization, C.P.C.; methodology, C.P.C.; software, Y.C.; validation, Y.C.; formal analysis, C.P.C.; investigation, Y.Y. and Y.L.; writing—original draft preparation, Y.Y., F.Y. and H.S.; writing-review and editing, C.P.C.; visualization, Y.C., Y.Y. and F.Y.; supervision, C.P.C.; project administration, G.L.; funding acquisition, G.L. All authors have read and agreed to the published version of the manuscript.

Funding: This research is funded by National Natural Science Foundation of China (61901264, 61831015), Science and Technology Commission of Shanghai Municipality (19ZR1427200), and Natural Science Foundation of Chongqing, China (cstc2021jcyj-msxmX1136).

Institutional Review Board Statement: Not applicable.

Informed Consent Statement: Not applicable.

Data Availability Statement: Data underlying the results presented in this paper are not publicly available but may be obtained from the authors upon reasonable request.

Acknowledgments: Special thanks to LightTrans International GmbH in Germany for offering the official license of VirtualLab Fusion.

Conflicts of Interest: The authors declare no conflict of interest.

\section{References}

1. Chen, C.P.; Zhou, L.; Ge, J.; Wu, Y.; Mi, L.; Wu, Y.; Yu, B.; Li, Y. Design of retinal projection displays enabling vision correction. Opt. Express 2017, 25, 28223-28235. [CrossRef]

2. Chen, C.; Li, H.; Zhang, Y.; Moon, C.; Kim, W.Y.; Jhun, C.G. Thin-film encapsulation for top-emitting organic light-emitting diode with inverted structure. Chin. Opt. Lett. 2014, 12, 022301. [CrossRef]

3. Chen, C.P.; Wu, Y.; Zhou, L.; Wang, K.; Zhang, Z.; Jhun, C.G. Crosstalk-free dual-view liquid crystal display using patterned E-type polarizer. Appl. Opt. 2017, 56, 380-384. [CrossRef] [PubMed]

4. Amitai, Y. Extremely compact high-performance HMDs based on substrate-guided optical element. In SID Symposium Digest of Technical Papers; Society for Information Display: Seattle, WA, USA, 23-28 May 2004; pp. 310-313.

5. Levola, T. Diffractive optics for virtual reality displays. J. Soc. Inf. Disp. 2006, 14, 467-475. [CrossRef]

6. Mukawa, H.; Akutsu, K.; Matsumura, I.; Nakano, S.; Yoshida, T.; Kuwahara, M.; Aiki, K. A full-color eyewear display using planar waveguides with reflection volume holograms. J. Soc. Inf. Disp. 2009, 17, 185-193. [CrossRef]

7. Wu, Y.; Chen, C.P.; Zhou, L.; Li, Y.; Yu, B.; Jin, H. Design of see-through near-eye display for presbyopia. Opt. Express 2017, 25, 8937-8949. [CrossRef] [PubMed]

8. Shi, Z.; Chen, W.T.; Capasso, F. Wide field-of-view waveguide displays enabled by polarization-dependent metagratings. Proc. SPIE 2018, 10676, 1067615.

9. Yoo, C.; Bang, K.; Chae, M.; Lee, B. Extended-viewing-angle waveguide near-eye display with a polarization-dependent steering combiner. Opt. Lett. 2020, 45, 2870-2873. [CrossRef]

10. Zhang, W.; Chen, C.P.; Ding, H.; Mi, L.; Chen, J.; Liu, Y.; Zhu, C. See-through near-eye display with built-in prescription and two-dimensional exit pupil expansion. Appl. Sci. 2020, 10, 3901. [CrossRef]

11. Chen, C.P.; Mi, L.; Zhang, W.; Ye, J.; Li, G. Waveguide-based near-eye display with dual-channel exit pupil expander. Displays 2021, 67, 101998. [CrossRef]

12. Vallius, T.; Tervo, J. Waveguides with Extended Field of View. U.S. Patent 9,791,703 B1, 13 April 2016.

13. Kress, B.C. Optical Architectures for Augmented-, Virtual-, and Mixed-Reality Headsets, 1st ed.; SPIE: Bellingham, WA, USA, 2020.

14. Guttag, K. Hololens 2 Display Evaluation Part 3: Color Uniformity. Available online: https://kguttag.com/2020/07/10/hololensdisplay-evaluation-part-3-color-uniformity (accessed on 7 November 2021).

15. Schott. SCHOTT RealView. Available online: https://www.schott.com/en-us/products/schott-realview-p1000268 (accessed on 6 November 2021).

16. Nys, I.; Stebryte, M.; Ussembayev, Y.Y.; Beeckman, J.; Neyts, K. Tilted chiral liquid crystal gratings for efficient large-angle diffraction. Adv. Opt. Mater. 2019, 7, 1901364. [CrossRef]

17. Ryabchun, A.; Bobrovsky, A. Cholesteric liquid crystal materials for tunable diffractive optics. Adv. Opt. Mater. 2018, 6, 1800335. [CrossRef]

18. Chen, C.P.; Su, Y.; Jhun, C.G. Recent advances in holographic recording media for dynamic holographic display. J. Opt. Photonics 2014, 1, 1-8. [CrossRef] 
19. Stebryte, M.; Nys, I.; Ussembayev, Y.Y.; Beeckman, J.; Neyts, K. Large angle forward diffraction by chiral liquid crystal gratings with inclined helical axis. Crystals 2020, 10, 807. [CrossRef]

20. Yin, K.; Zhan, T.; Xiong, J.; He, Z.; Wu, S.-T. Polarization volume gratings for near-eye displays and novel photonic devices. Crystals 2020, 10, 561. [CrossRef]

21. Mi, L.; Chen, C.P.; Lu, Y.; Zhang, W.; Chen, J.; Maitlo, N. Design of lensless retinal scanning display with diffractive optical element. Opt. Express 2019, 27, 20493-20507. [CrossRef] [PubMed]

22. Yeh, P.; Gu, C. Optics of Liquid Crystal Displays, 2nd ed.; Wiley: Hoboken, NJ, USA, 2009. 